\title{
PRIMARY CEMENTLESS TOTAL HIP ARTHROPLASTY IN ANKYLOSING SPONDYLITIS
}

\author{
K. Nageshwara Rao ${ }^{1}$, Ravikumar Gudipati²
}

${ }^{1}$ Assistant Professor, Department of Orthopaedics, Nizam's Institute of Medical Sciences.

${ }^{2}$ Senior Resident, Department of Orthopaedics, Nizam's Institute of Medical Sciences.

ABSTRACT
INTRODUCTION
Ankylosing Spondylitis (AS), family of Spondyloarthritides (SpAs), is a chronic inflammatory disease affecting the axial skeleton,
the entheses and occasionally the peripheral joints. The shoulders and hips are considered axial joints and involvement occurs in up
to $50 \%$ of patients and is more common than involvement of the more distal joints. Aim of our study is to evaluate outcome of
Cementless Total Hip Arthroplasty (THA) in ankylosing spondylitis.

\section{MATERIALS AND METHODS}

We prospectively and retrospectively reviewed 27 hips in 20 patients who underwent cementless THA between 2007-2013. Mean age of patient was 31.5 years. We analysed demographic data, preoperative deformity, Harris Hip Score, ambulatory status, need for walking aids.

\section{RESULTS}

All patients experienced significant improvement in function, range of motion, posture and ambulation. Postoperative Harris Hip Score improved from 18.95 to $89.35 ; 90 \%$ are completely pain free, 5\% have occasional discomfort and 5\% have mild-to-moderate pain.

\section{CONCLUSION}

Cementless THA for deformed hips in young patients with AS is worthwhile surgical intervention, as it increases the mobility of the patient, improves the ability to sit comfortably, decrases the morbidity of the disease. However, the technically demanding nature of the procedure should not be underestimated.

\section{KEYWORDS}

Cementless total hip arthroplasty, ankylosing spondylitis.

HOW TO CITE THIS ARTICLE: Rao K. N, Gudipati R. “Primary cementless total hip arthroplasty in ankylosing spondylitis.” Journal of Evolution of Medical and Dental Sciences 2015; Vol. 4, Issue 103, December 24; Page: 16846-16849,

DOI: $10.14260 /$ jemds/2015/2531

\section{INTRODUCTION}

Ankylosing Spondylitis (AS), family of Spondyloarthritides (SpAs), is a chronic inflammatory disease affecting the axial skeleton, the entheses and occasionally the peripheral joints. The shoulders and hips are considered axial joints and involvement occurs in up to $50 \%$ of patients and is more common than involvement of the more distal joints. In the hips, there may be progressive flexion deformity and eventual destruction of the joint. Destructive changes in the hips are slowly progressive and associated with marked deformity of the femoral head before the development of ankyloses. Hip disease in AS is a marker of more severe disease in adults. There is a higher prevalence of hip involvement and greater need for hip arthroplasty in juvenile-onset AS. ${ }^{1}$ Ankylosis of the hip can cause neighbouring joint pain, such as low back pain and knee pain.

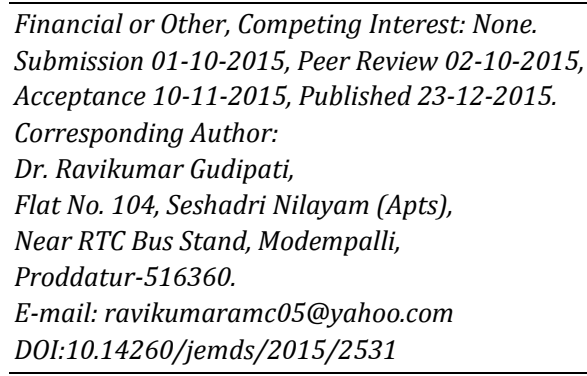

The results and the benefits of this conversion have been reported since the 1970s. ${ }^{2}$ but the procedure has been described as technically difficult.2-5 As these patients posses several problems like severe deformities of knee with bony ankylosis that results in difficulties in positioning, difficulty in giving anaesthesia due to fused spine, need for adductor tenotomy and release of other muscles around the hip, need for special implants like acetabular reinforcement rings, unpredictable gain in ROM, heterotopic ossification and influence of drug therapy (steroids and DMARDS).

The results of cemented THA in patients with advanced symptomatic disease of the hip in AS have been reported.6,7 Only a few reports, however, concerning the use of cementless THA in ankylosing spondylitis has been published.8,9 Our study is retrospective observational study to evaluate the clinical and radiological outcome of cementless THA in ankylosing spondylitis patients.

\section{MATERIALS AND METHODS}

We reviewed 20 patients who underwent THA in AS between 2007-2013 with longest followup of 7 years and shortest followup of 10 months. We analysed demographic data, preoperative deformity, Harris Hip Score, ambulatory status, need for walking aids before and after surgery. All patients met the diagnostic criteria of Ankylosing Spondylitis and had been followed by a rheumatologist on a routine basis. The main indication for surgery was ankylosis of hip with poor posture. Disabling pain with poor posture was the indication for hip 
arthroplasty in some patients. We have analysed the hospital records of all the 20 patients who came for followup. The records were analysed with regards to associated risk factors, medical illness, pre-op, per-op, post-operative complications, till the patient was discharged and regularly followed in outpatient department.

Fifteen cases were operated under combined spinal epidural anaesthesia and 5 cases under general anaesthesia with the help of fibreoptic bronchoscope in 2 patients. An anterolateral approach of Hardinge was used in all the cases. Supine position of the patient provides a good opportunity to evaluate the orientation of the acetabulum. During acetabular preparation, we always tried to identify the margin of the acetabulum before reaming was begun. In patients with bony ankylosis, osteotomy of the neck of the femur was done in situ with a power saw and reaming was done until pulvinar tissue is seen. (Figure 1, 2, 3).

Heparin (LMW) was given subcutaneously as thromboprophylaxis for 7 days.

Prophylaxis against heterotopic bone formation was not given in any of our cases.

Patients were kept on in-bed mobilization for 6 weeks and then gradually progressed to weight-bearing and walking over the next six to eight weeks. Patients who underwent cerclage wiring of the femur, extended trochanteric osteotomy or had intraoperative fractures were subjected to an average of 12 weeks of non-weightbearing.

We used cementless metaphyseal porous coated (SUMMIT; Depuy) stem in 10 hips and Hydroxyappetite coated stem (CORAIL; Depuy) in 17 hips. Cementless DUROLAC (Depuy) acetabular shell used in 25 hips and PINNACLE (Depuy) used in two hips.Highly crosslinked polyethelene bearing surfaces used in 25 hips and METAL ON METAL used in 2 hips, adjunctive fixation was achieved with screws in all these cases.

All patients were contacted through telephone and followed in outpatient department for follow-up between August 2012 and September 2014.

Clinical assessment at follow-up include assessment of the pain, function, deformities and range of motion using the Harris Hip Score. ${ }^{10}$ is evaluated. The sum range of motion was measured. This refers to the sum of flexion, abduction, adduction, internal and external rotation movements achieved by the patient. Radiographic evaluation comprising anteroposterior radiographic views of the pelvis and femur and a lateral view of the femur were performed at follow-up visits. The femoral stems were evaluated radiographically using the system of Engh et al. ${ }^{11}$ The bone prosthesis interface was evaluated for radiolucencies and recorded according to the zonal system described by Gruen et al.12 Acetabular evaluation was performed using the criteria described by DeLee and Charnley. ${ }^{13}$ Acetabular inclination was measured using the interteardrop line as a reference point. ${ }^{14}$ Ectopic ossification was graded according to the system described by Brooker et al..$^{15}$

\section{RESULTS:}

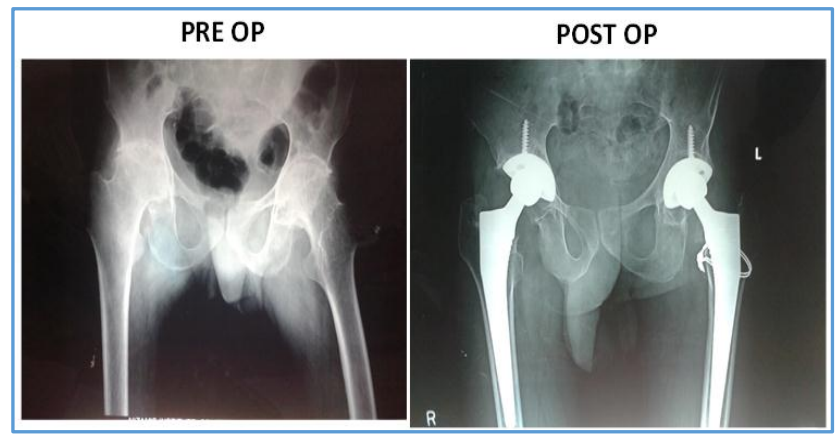

All the patients met the diagnostic criteria of AS. We analysed 20 patients in our study, of those 18 are males and 2 are females. Bilateral THR done in 7 patients. The average preoperative Harris Hip Score was 18.95 points. At the most recent followup, the average post-operative Harris hip score was 89.35 points. The Harris hip scores improved over a period of time. The result was excellent in 15 patients, good in 2 patients, fair in 2 patients, poor in 1 patient. There was marked immediate relief in pain postoperatively. After improving for three months, however, the pain scores did not change appreciably; $18 / 20(90 \%)$ patients were totally pain free; $1 / 20(5 \%)$ patients had slight occasional pain without compromising daily activities; $1 / 20(5 \%)$ patients had moderate pain, some limitation of ordinary work. Mild anterior thigh pain was present in one of our cases. Slight limp was present in $8 / 20(40 \%)$ patients without compromising daily activities. Moderate limp was present in $1 / 20(5 \%)$ patients; $15 / 20(75 \%)$ patients walked without support; $2 / 20(10 \%)$ required a cane for long walks; $3 / 20(15 \%)$ required cane most of the time; $16 / 20(80 \%)$ patients could walk unlimited distance; $1 / 32(5 \%)$ patients could walk six blocks; $2 / 20(10 \%)$ patients could walk two to three blocks; $1 / 20(5 \%)$ patients confined to indoors only because increased severity of the disease. ALL patients were able to use public transportation. Limb Length Discrepancy (LLD) was restored to not more than $1 \mathrm{~cm}$ in 19 cases. One patient had significant limb length discrepancy, which was managed with shoe rise. All our patients were satisfied with the outcome of their operation. All the patients considered hip to have better function than prior to surgery.

\section{Radiographic Outcome}

Radiographic assessment revealed consistent evidence of bony ingrowth.

All hips showed signs of osseous ingrowth (fixation/stability score +10 ). The femoral stem was aligned in neutral in 22 hips (95.60\%). One hip was aligned in slight valgus. Metaphyseal fit was good in all the cases. Resorptive bone remodeling like mottling or cortical thinning was not seen. Endosteal spot welds were seen in $50 \%$ of the cases. Pedestal formation was not seen in any cases. Serial radiographs showed no evidence of subsidence, migration or bead shedding; 5 cups had radiolucent lines which were nonprogressive. No hip had radiolucent line more than $2 \mathrm{~mm}$. None of the screws were broken. No radiolucent lines were seen around the porous surfaces.

All acetabular components were stable. Average acetabular inclination was 40 degrees. Superior coverage was $>95 \%$ in all the cases.

Three patients developed intraoperative hoof stress crack fractures for which circlage wiring was done, which healed and patients were under the standard postop rehabilitation. Patients had excellent clinical and radiological scores at last followup.

Not more than one patient developed Brooker class I heterotopic ossification, which was insignificant.

None of the patients had dislocation.

\section{DISCUSSION}

Only few reports of total hip arthroplasty in patients with ankylosing spondylitis are available. They dealt not only with patients with ankylosing spondylitis, but also with rheumatoid arthritis patients and they did not separate their results according to the disease process.

Our main indication for surgery in ankylosing spondylitis, in which there is involvement of the hips is poor posture because of the ankylosed hips or the presence of the painful arc of motion and limitation of ambulation or both. The stooped position of patients with ankylosing spondylitis may 
be caused by severe hip-flexion contracture or kyphosis of the thoracolumbar spine or both. Correcting the hip-flexion contracture with total hip replacement yields a significant improvement in posture - all of our patients had significant improvement in posture. Patients with bilateral hip-flexion contracture did best when arthroplasty was performed bilaterally. In three patients with bilateral flexion contracture, only one arthroplasty was done. The improvement in posture in these patients was not as satisfactory as one would like, because of persistent flexion contracture of the side not operated on. One patient had excision arthroplasty on one side done at another hospital and he had significant limp.

Loss of hip movement is most marked in ankylosing spondylitis, where hip muscles have sometimes been inactive for a number of years. Consequently the range of movements following surgery may be disappointing, although pain is relieved, but in the long term these patients also have good functional improvement. In bone ankylosis the limited gain in motion is due to long-standing hip disease, poor muscles, myositis ossificans and the basic nature of the disease.

Ambulation and functional ability improved in all patients. There was a good correlation between the sum total range of motion and abductor power. Late mechanical failures may become evident in long-term follow-up of these young patients, because patients with ankylosing spondylitis are more active. In addition, an ankylosed spine may impose excessive strain on the artificial joint causing late failure of the prosthesis. Long-term durability can be assessed only after longer follow-up.

\section{Clinical Outcome}

In our series, the postoperative clinical hip scores compared favourably to the scores reported earlier in the literature.

In our series 15 patients had excellent Harris hip scores, 2 had good scores and fair score in 2 hips and poor in 1 hip similar to most previous studies. ${ }^{[3-5]}$ with uncemented as well as cemented arthroplasties. No deterioration of hip scores has been seen during the followup. The Harris Hip Scores improved over a period of time. Limp was present in 9 patients $(45 \%)$ as compared to $28 \%$ in Callaghan study. ${ }^{16}$ Rates of limp in our study was higher as compared to Callaghan's study. We attribute this to the multiple joint involvement with long duration of disease and extensive joint involvement preoperatively. We started a vigorous abductor strengthening exercises postop. ONE patient with ankylosing spondylitis had bilateral thigh pain $(5 \%)$ in our study, which was less as compared to $18 \%$ incidence of thigh pain reported by Callaghan. ${ }^{16}$ 20\% (Dodge), Engh. ${ }^{11}$ reported a 14\% incidence of thigh pain that was activity related. The pain in the thigh was more of muscle strain and there were no signs of instability of implant in the patient. In our study, most common site for the endosteal bone bridging (Spot welds) was in the Gruen zone.12 1 and 7 where the stem is porous coat.

Preoperatively planned sizes of the acetabular cup and the femoral stem by templating showed a high rate of coincidence with the sizes used intraoperatively in our series. So accurate and careful preoperative templating can result in a balanced hip reconstruction by correcting the leg length differences and restoring the offsets. The most common cup size that we used was $52 \mathrm{~mm}$. The common stem size was between 5 and 7. In our series, all the femoral stems were in neutral except for one which was in slight valgus. Our results are comparable with the recent reports. ${ }^{[8,9]}$ with improved implant designs with second generation tapered hip stems. In our series, 95.6\% hips showed signs of osseous ingrowth. No signs of implant instability were found. These results are consistent with previous results with Cementless hips. $5,17,8,9,6,7$
The signs of fixation described by Engh et al. ${ }^{11}$ that is spot weld formation and the absence of lines in the porous coated region showed perfect correlation with durable fixation in our series.

Only one patient $(<5 \%)$ developed Brooker class I heterotopic ossification even without any prophylaxis as compared to studies, which shows $5-80 \% .18$ This may be due to chronic NSAIDs intake in these patients, meticulous surgical technique and early ambulation.

Total hip replacement has now been shown to provide rapid and reliable pain relief in patients with Ankylosing spondylitis. Range of movement and function are significantly increased and all of the benefits are well maintained in the long term. The complication rate is low and could be improved further by anticipation and meticulous preoperative planning. Component survivorship has been shown to be comparable with other patient groups when using a tried and tested implant. However, dealing with these young, active and highly motivated patients is often both emotionally demanding and technically challenging and such cases should only be operated upon by experienced hip surgeons. High patient satisfaction may be anticipated in most cases, provided the clinician uses, sound clinical judgment, properly assesses patient's goals and expectations, and communicates realistic expectations to the patient.

\section{CONCLUSION}

In our patients total hip arthroplasty provides excellent pain relief, had good functional outcome. Controversy continues with regard to the best mode and technique of fixation, implant design and articulating surfaces. Excellent results are possible with a number of designs. We conclude that with porous coated and hydroxyapatite coated cementless stems with good initial stability, high rates of clinical success were attained in these patients with ankylosing spondylitis. Incidence of heterotopic ossification can be reduced with meticulous surgical technique and there is no need of any prophylaxis. Most of our patients are young active patients with high functional demands and have shown excellent clinical and radiological results. But we may need furthur long term follow-up for better evaluation. THA in these patients increases the mobility of the patient, improves the ability to sit comfortably, decreases the morbidity of the disease. However, the technically demanding nature of the procedure should not be underestimated.

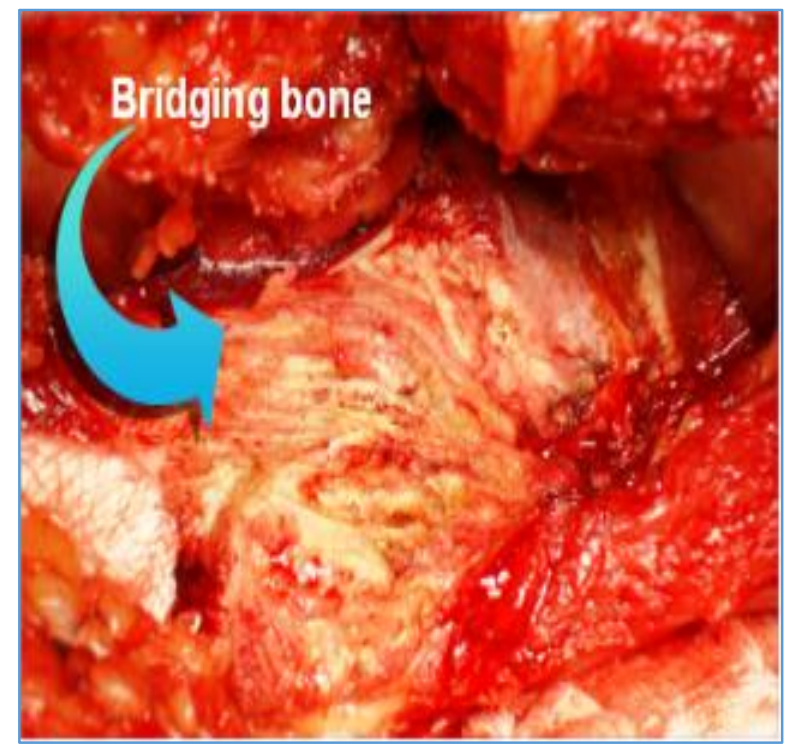

Bridging bone between Femur and Acetabulum 


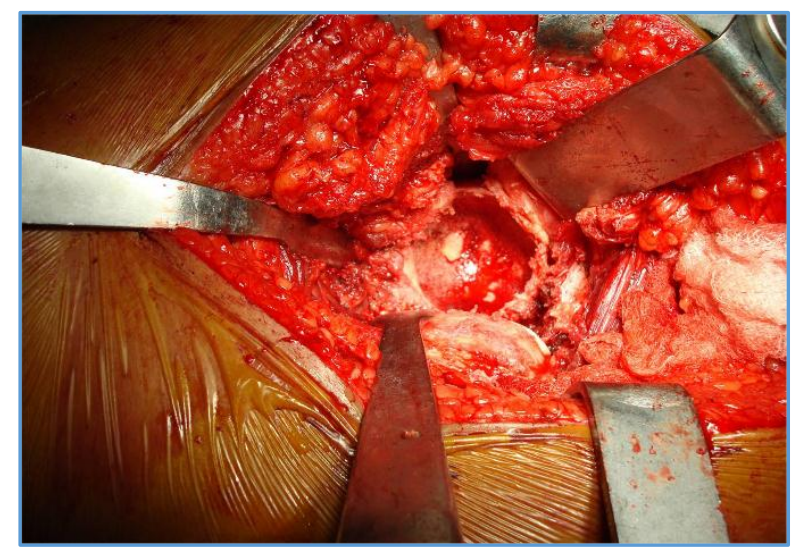

Creating Acetabulum

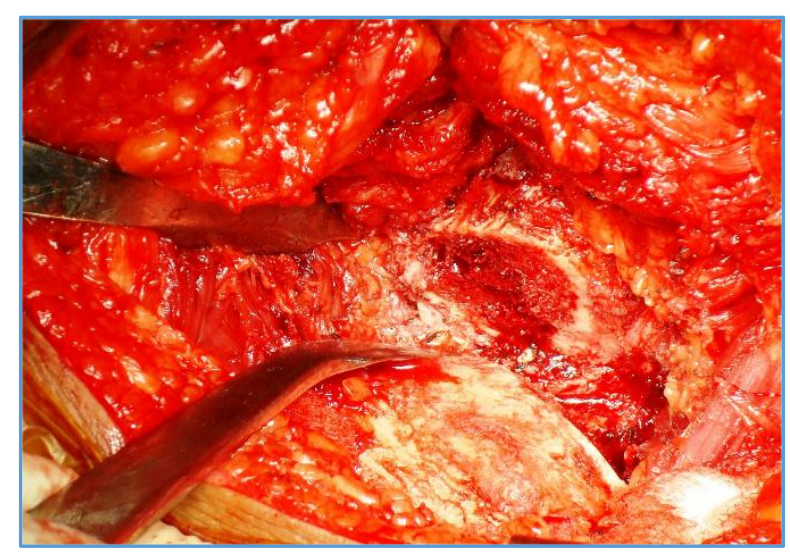

Neck In Situ Osteotomised

\section{REFERENCES}

1. Marc C Hochberg, MD, MPH; Alan J Silman, MD, MSC, FRCP, FMedSci., Rheumatology, $5^{\text {th }}$ edition 2011 page no. 1129.

2. Amstutz HC, Sakai DN. Total joint replacement for ankylosed hips: indications, technique and preliminary results. J Bone Joint Surg [Am] 1975;57:619.

3. Kilgus DJ, Amstutz HC, Wolgin MA, et al. Joint replacement for ankylosed hips. J Bone Joint Surg [Am] 1990;72:45.

4. Strathy GM, Fitzgerald RH. Total hip arthroplasty in the ankylosed hip. A ten-year follow-up. J Bone Joint Surg [Am] 1988;70:963.

5. Kim YH, Oh SH, Kim JS, et al. Total hip arthroplasty for the treatment of osseous ankylosed hips. Clin Orthop 2003;414:136.
6. Jia Li, MD; Weidong Xu, MD; Ling Xu, MD and Zhimin Liang, MD. Hip Resurfacing Arthroplasty for Ankylosing Spondylitis. The Journal of Arthroplasty Vol. 24 No. 82009.

7. Long-Term results of total hip replacementin young patients who had ankylosing spondylitis eighteen to thirtyyear results with survivorship analysis by david $h$ Sochart, frcsf and martyn l Porter, frcs 4, wiganjbjsvol. 79-a, no. 8, august 1997.

8. YongLae Kim, MD; Sang Ik Shin, MD; Kwang Woo Nam, MD, et al. Total hip arthroplasty for bilaterally ankylosed hips. The Journal of Arthroplasty Vol. 22 No. 7, 2007.

9. Surya Bhan, MS, FRCS(Ed.); Krishna Kiran Eachempati, MS; and Rajesh Malhotra, MS. Primary Cementless Total Hip Arthroplasty for Bony Ankylosis in Patients with Ankylosing Spondylitis. The Journal of Arthroplasty Vol. 23, No. 6, 2008.

10. Harris WH. Traumatic arthritis of the hip after dislocation and acetabular fractures: treatment by mold arthroplasty: an end-result study using a new method of result evaluation. J Bone Joint Surg Am 1969;51:737.

11. Engh CA, Massin P, Suthers KE. Roentgenographic assessment of the biologic fixation of porous-surfaced femoral components. Clin Orthop 1990;257:107.

12. Gruen TA, McNeice GM, Amstutz HC. "Modes of failure" of cemented stem-type femoral components: a radiographic analysis of loosening. Clin Orthop 1979;141:17.

13. DeLee JG, Charnley J. Radiological demarcation of cemented sockets in total hip replacement. Clin Orthop 1976;121:20.

14. Brinker MR, Rosenberg AG, Kull L, et al. Primary noncemented total hip arthroplasty in patients with ankylosing spondylitis, Clinical and radiographic results at an average follow-up period of 6 years. J Arthroplasty 1996;11:802.

15. Brooker AF, Bowerman JW, Robinson RA, et al. Ectopic ossification following total hip replacement. Incidence and a method of classification. J Bone Joint Surg [Am] 1973;55:1629.

16. Callaghan, Dysart JJ. The uncemented porous coated anatomic total hip prosthesis J Bone and Joint Surg 1988;70A:3.

17. Hamadouche M, Kerboull L, Meunier A, et al. Total hip arthroplasty for the treatment of ankylosed hips: a five to twenty-one-year follow-up study. J Bone Joint Surg [Am] 2001;83:992.

18. Dhaon BK, Anuj Jaiswal, Vishal Nigam, et al. Non-cemented total hip replacement in various disorders of the hip. Indian Journal of Orthopaedics, October 2005, Volume 39: Number 4: P. 225-227. 